\title{
Prototyp eines mobilen Warenausgangs- und Liefersystems
}

\author{
Ralf Vandenhouten, Miriam Selz
}

\section{Einleitung}

\subsection{Motivation und Zielstellung}

Der zu erwartende Wandel des gesamten Marktes in den nächsten Jahren - insbesondere durch den Einsatz von eCommerce und mCommerce - macht auch eine Anpassung der Unternehmen und ihrer Logistik erforderlich. Der Einsatz moderner Informationstechnologie (IT) erlaubt die Optimierung von Prozessen und einen verbesserten Kundenservice, wie z. B. die Verfolgung von Paketen, die heute bei kleinen und mittleren Speditionen noch nicht zum Standard gehört. Durch den Einsatz von Warenwirtschaftssystemen mit Barcodeintegration wird in Großhandelsunternehmen mit eigenem Fuhrpark eine Paketverfolgung nur bis zur Laderampe, also „Inhouse“, gesichert. Die weiterführende Verfolgung der Sendungen fehlt jedoch. In größeren Logistikunternehmen ist, durch Ausstattung der Nutzfahrzeuge mit relativ aufwändiger Bordelektronik, das Tracking und Tracing der Versandeinheiten zwar weitestgehend gesichert. Verbesserungspotential liegt hier aber unter anderem in der Disposition und im Tracking der Transporthilfsmittel (Gitterboxen, Paletten), die zurzeit in der Regel noch nicht systematisch erfasst und verfolgt werden.

Ziel des hier vorgestellten Vorhabens war die Entwicklung eines mobilen Warenausgangs- und Liefersystems, das durch den konkreten Bedarf eines Unternehmens im Elektrogroßhandel (im Folgenden als „Pilotunternehmen“ bezeichnet) motiviert wurde. Damit sollte, in Ergänzung zum bestehenden Warenwirtschaftssystem, eine Sendungsverfolgung über die Laderampe hinaus sowie ein Tracking der Transporthilfsmittel ermöglicht werden. Um die Kosten-Nutzen-Relation einer solchen Implementierung zu rechtfertigen, sollte das System keine Komponenten beinhalten, die nicht benötigt werden. Auf dem Markt erhältliche mobile Systeme wurden zum

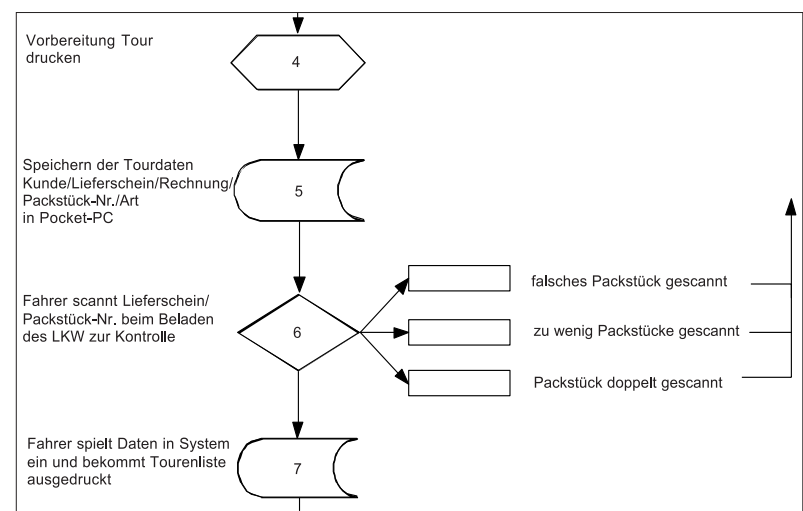

Abb. 1a: Auszug der Geschäftsprozessanalyse, Beladung

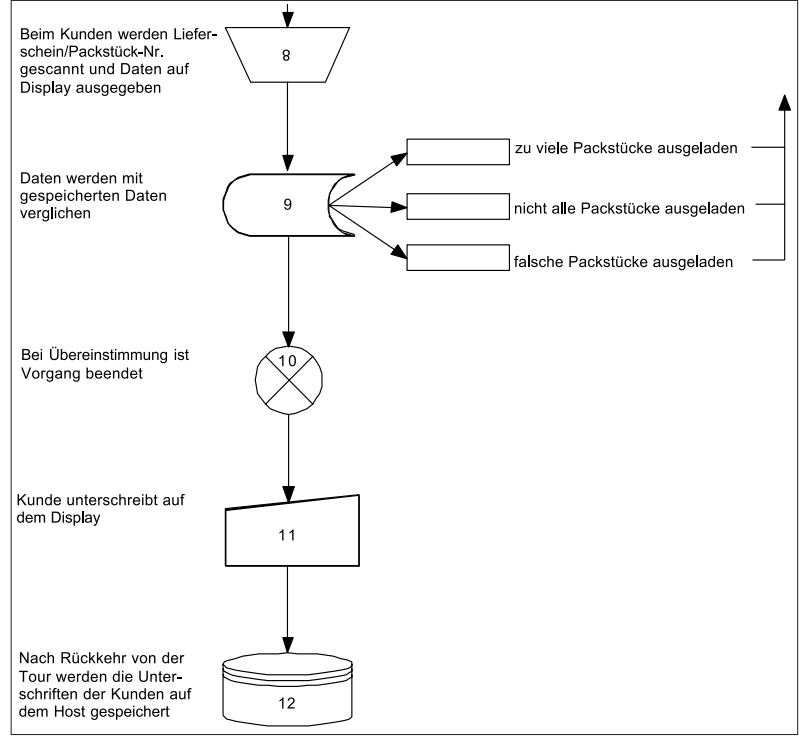

Abb. 1b: Auszug der Geschäftsprozessanalyse, Belieferung

einen einzelnen Anforderungen des Unternehmens nicht gerecht und waren zum anderen zu komplex (und damit zu kostenintensiv) für die gegebene Aufgabenstellung, so dass sich das Unternehmen für eine individuelle Realisierung entschied.

Eine Geschäftsprozessanalyse verdeutlichte, welche Prozesse bei der Gestaltung des Konzeptes betrachtet werden mussten. Im Vordergrund standen dabei die logistischen Vorgänge innerhalb des Warenausgangs und der Lieferung. Die Abbildungen 1a und 1b stellen einen Auszug der zu optimierenden Prozesse dar.

Das zu entwickelnde System sollte einerseits die Abläufe im Warenausgang (Warenausgangskontrolle, Versandabwicklung, Auslieferung) optimieren. Innerhalb der Disponierungsvorgänge wurde eine Optimierung des Dokumentenhandlings angestrebt. So sollten das manuelle PapierHandling und die manuelle Archivierung der Lieferdokumente möglichst entfallen. Ziel war deshalb die Erhöhung des beleglosen Geschäftsverkehrs durch Digitalisierung der Dokumente (Lieferschein, Retourenschein, Rechnung der Scheck- bzw. Barzahler) und der Kundenunterschrift. Der Beladungs- und Entladungsvorgang schließlich sollte durch die Einführung mobiler Barcode-Scan-Technologie sicherer, schneller und komfortabler werden.

\subsection{Ausgangssituation}

Bei dem Pilotunternehmen handelt es sich um einen Großhändler in der Elektronikbranche, der in Deutschland uiber mehrere Standorte verfuigt. 
Der Prototyp wurde am Standort Ludwigsfelde (Logistikzentrum mit Lager) realisiert. Mit dem firmeneigenen Fuhrpark, bestehend aus 28 Lkws (1 bis 2 Fahrer je Lkw), werden täglich bis zu 750 Kunden und 22 Filialen angefahren.

Das gesamte Lager besteht aus vier Lagerteilen:

- manueller Bereich: Kapazität 1600 Paletten

- Trommellager (Freifläche): Kapazität: 1300 Kabel-Trommeln

- Hochregallager: Kapazität: 5261 Paletten; Bewegung: ca. $10 \%$

- Automatisches Kleinteillager: Kapazität: 36187 Behälter; Bewegung ca. $80 \%$

Die Kommissionierung erfolgt halbautomatisch. Das bedeutet, dass die Warenentnahme teils manuell durch das Lagerpersonal und zum Teil durch Regalbediengeräte durchgefuihrt wird. Das Hochregallager sowie das Kleinteillager sind weitestgehend durch automatische Regalbediengeräte, welche die entsprechenden Lagerplätze mit Hilfe der computergestuitzten Lagerverwaltung automatisch auf optimierter Linearfahrt ansteuern, und durch kabelgebundene Scannertechnik (inkl. BarcodeSystem) automatisiert.

Nach Auftragseingang (Telefon, Fax, Internet) und der Kommissionierung erfolgt die Vorbereitung der Tour. Dieses Szenario wird wie folgt gehandhabt: Die Dokumente (Lieferscheine, Rechnungen, Retourenscheine) werden fuir alle Fahrer ausgedruckt. Anhand der Postleitzahlen auf den Dokumenten sortiert jeder Fahrer seine Tour. Danach scannt der Disponent die sortierten Lieferscheine ein, so dass auch das Warenwirtschaftssystem die Tourenpläne der Fahrer erhält und der Disponent bei Anfrage eine ungefähre Aussage über die Lieferzeit geben kann. Danach belädt der Fahrer den Lkw an der zugeteilten Laderampe mit der auf den Lieferscheinen befindlichen Ware und fährt seine Tour ab. Nach der Tour werden die unterschriebenen Lieferscheinkopien vom Fahrer abgeliefert und in Ordnern archiviert.

\subsection{Probleme und Anforderungen}

Besonders leidet das Unternehmen an den Folgen des manuellen Dokumentenhandlings (Ausdruck, Kopien abheften). Es besteht ein riesiges Archivierungslager mit hohen Lohn- und Lagerkosten. Damit die Zentrale Einsicht in die Tourenpläne hat, muss der Disponent alle vom Fahrer sortierten Lieferscheine (bis zu 50 pro Tour) einscannen.

Die Liefereinheiten verschwinden ab der Laderampe aus dem Kontrollmechanismus. An der Laderampe kommt es häufig zur Falschbeladung des Fahrzeuges, da u. a. die Waren manuell zusammengestellt werden.

Die Verfolgung der Verpackung (Paletten, Gitterboxen, Plastikboxen) ist ausschließlich durch die Angabe der Verpackungsart auf dem Lieferschein gegeben. Der Verbleib der Verpackung bleibt außen vor (Kosten durch Verlust: je Palette ca. 10 bis 30 Euro).

Mit der Einfuihrung des rechnergestuitzten, mobilen Warenausgangs- und Liefersystems waren einige qualitative
Nutzenserwartungen verbunden. Es sollte ein mobiles Terminal zum Einsatz kommen. Klein und leicht sollten die Geräte sein, das Touchdisplay und der Scanner gut bedienbar und robust für den Einsatz in Zustellfahrzeugen. Der Scannvorgang sollte schnell abgewickelt werden können und die Fahrer sollten beim Tragen der Pakete nicht behindert werden. Die mit einem Barcode versehenen Liefereinheiten und Lieferdokumente (Lieferscheine, Rechnungen der Bar-/Scheckzahler) sollten eingescannt werden können. Die Lösung sollte es dem Fahrer ermöglichen, mobil auf die Lieferinformationen seiner Tour zugreifen zu können. Es sollte auch die Möglichkeit bestehen, eine digitalisierte, sichere Bestätigung der Auslieferung vom Kunden abspeichern zu können, d. h. die belieferten Kunden sollten auf dem mobilen Gerät unterschreiben können. Die Kundenunterschrift und die Lieferscheindaten sollten in der Datenbank archiviert werden können und jederzeit wieder abrufbar sein.

Zudem sollte die Tourenplanerstellung optimiert bzw. erleichtert werden, ohne in den üblichen Sortiervorgang einzugreifen.

\subsection{Kriterien und Planungsgrundlagen}

Voraussetzung für die Planung waren folgende Anforderungskriterien:

- Anwenderfreundliche Oberfläche des mobilen Gerätes (Bedienung weitgehend mit dem Finger).

- Jedes mobile Gerät hat eine Tagestour gespeichert. Die Tour wird anhand der elektronischen Lieferscheine auf dem jeweiligen Endgerät angezeigt.

- Soll-Ist-Abgleich: Durch Scannfunktion des mobilen Gerätes und entsprechender Applikation, die den Barcode lesen kann, soll möglichst eine durchgängige Kontrolle der Liefereinheiten vom Lager (Laderampe) bis zur Auslieferung beim Kunden erreicht werden.

- Erfassung von Retouren (manuelle Bestätigung ohne Scanner).

- Verfolgung von Paletten und Boxen (Verpackungsarten) durch manuelle Eingabe.

- GUI für das Depotpersonal für die Datenübertragung (einsetzbar in jeder Filiale).

- Digitale Übermittlung der Tourendaten (Lieferschein) aus der Datenbank auf das mobile Endgerät.

- Der Zugriff auf die Daten soll uiber einen Arbeitsplatzrechner möglich sein.

- Übermittlung der Ruicklaufdaten, inklusive der digitalen Bestätigung des Kunden, zur Archivierung in die Datenbank.

\section{Planung und Entwicklung des Systems}

Das System sollte durch die Scannvorgänge und die zusätzlichen manuellen Eingabemöglichkeiten am mobilen Gerät einer verbesserten Waren- und Verpackungsverfolgung dienen. Zudem sollte es den Prozess der Tourenplanung erleichtern, die Beladung des Lkws an der Laderampe sicherer abhandeln, eine (auftragnehmerseitig) beleglose Lieferung ermöglichen und die digitalen und unterschriebenen Lieferdokumente archivieren. 


\subsection{System-Voraussetzungen/} Hardware- und Softwarebasis

1. 30 PDAs der Firma Symbol:

- mit LCD-Display (Touchscreen)

- integrierter Barcode-Scanner mit Laser-Rotationsspiegel-Technik

- Betriebssystem: Microsoft Windows mobile 2003 for PocketPCs, JVM (Java Virtual Machine) inkl. Scannertreiber fuir die PDAs

- Kommunikation: WLAN (Wireless Lokal Area Network), Standard IEEE802.11b

2. notwendige Hardware-Komponenten zur Datenuibertragung: Access-Points für WLAN-Netz

3. Web- und Applikationsserver (Betriebssystem SuSE Linux 9.0)

4. browserfähige Workstations (Windows 2000/XP oder Linux)

Das System basiert auf einer Client-Server-Architektur. Die Server-Komponente läuft auf einem Linux-Rechner, die PC-Client-Komponente auf gängigen browserfähigen Windows-Arbeitsplatzrechnern. Die mobile Komponente des PDA ist unter dem PDA-Betriebssystem MicrosoftWindows mobile 2003 und einer JVM (Java Virtual Machine) ablauffähig.

Die Datenhaltung erfolgt in der firmeneigenen Datenbank auf einem Mainframe-Rechner (IBM AS/400). Über LAN (TCP/IP) erfolgt der Zugriff vom Applikationsserver auf die Daten in der Firmendatenbank.

Die Kommunikation zwischen Applikations-Server und den mobilen Endgeräten erfolgt über WLAN.

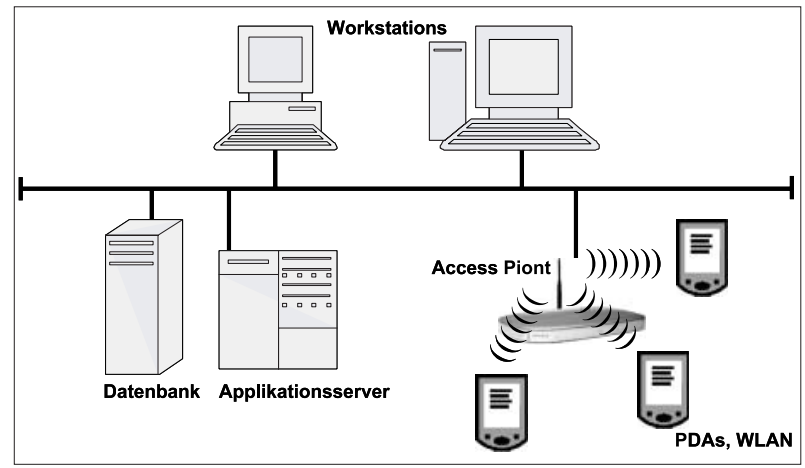

Abb. 2: Netzwerkstruktur

\subsection{Architektur}

\subsubsection{Clients}

Über das Internet (Webserver) haben die Workstations (Client-PCs) des Systems von uiberall Zugriff auf die Synchronisationsapplikation. Die Applikationslogik wird auf dem Web-Server ausgefuihrt (Servlets und Java Server Pages). Lokal auf den Client-Rechnern wird nur ein gewöhnlicher Internet-Browser (z. B. Mozilla oder Internet-Explorer) benötigt. Die PDA-Clients werden ins interne WLAN eingebunden.

\subsubsection{Applikationsserver}

Die Architektur des Applikationsservers verwendet ein Drei-Schicht-Modell (Webapplikationsschicht, Schicht für die Fachlogik und Datenbankzugriffsschicht). Ein Kom-

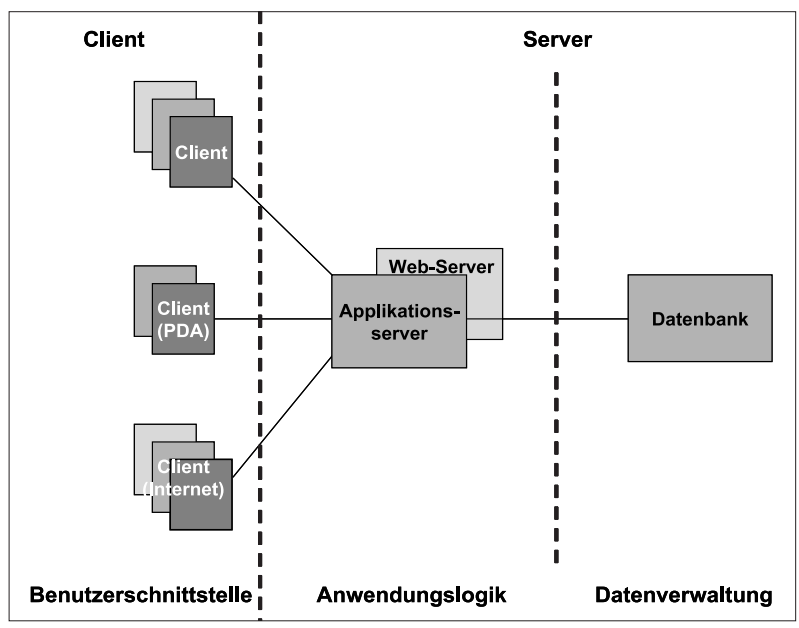

Abb. 3: Software-Architektur des Systems

munikations-Softwaremodul regelt die Kommunikation zwischen dem Server und dem DB-System und die Kommunikation zwischen den Web-Clients und dem System. Java Servlets kommunizieren über eine definierte Schnittstelle mit dem Server. Mit Hilfe des JDBC-API (Java Database Connectivity) wird der Zugang der Fachlogikmodule zur lokalen SQL-Datenbank des Applikationsservers ermöglicht.

\subsubsection{Software}

Bei der Softwareentwicklung wurden konsequent modulare Konzepte umgesetzt. Die zum größten Teil in Java entwickelten Softwarekomponenten ermöglichen die Wiederverwendung von Teilen der Software und steigern dadurch die Effizienz im Entwicklungsprozess. Weitere Vorteile sind die Flexibilität des Systems durch Adaptierbarkeit, Skalierbarkeit und klar definierte Schnittstellen sowie die Wartbarkeit des Systems.

Die Softwaremodule fuir den Zugriff auf die unternehmensinterne Infrastruktur sind als Middleware eingebunden. Zu den Aufgaben gehört die automatische Datenkonvertierung als auch die Abwicklung des Datenflusses bzw. die Bereitstellung entsprechender Schnittstellen.

\section{Synchronisationssoftware}

Die Software für die Disposition konnte auf dem Applikationsserver implementiert werden. Dies macht eine eigene Intelligenz der PC-Clients überflüssig. Diese Anwendung sollte in der Lage sein, die Funktion des Ein- und Auslesens (neuer) Daten zum bzw. vom PDA auszulösen und den aktuellen Status der PDAs auf dem Client-PC zu visualisieren.

Die Synchronisationssoftware verwendet das XML-Format (eXtensible Markup Language) für den Austausch von Daten, das sich inzwischen als Standard etabliert hat. XML ist eine textbasierte Meta-Auszeichnungssprache, wurde vom W3C (World Wide Web Consortium) entwickelt und 1998 als offener Standard veröffentlicht. XML ermöglicht es, Daten bzw. Dokumente so zu beschreiben und zu strukturieren, dass sie zwischen einer Vielzahl von Anwendungen ausgetauscht und weiterverarbeitet werden können, insbesondere über das Internet. Abbildung 4 zeigt, welche Zustände in der Synchronisationssoftware durch Auswertung der übertragenen Steuerbefehle angenommen werden können. 


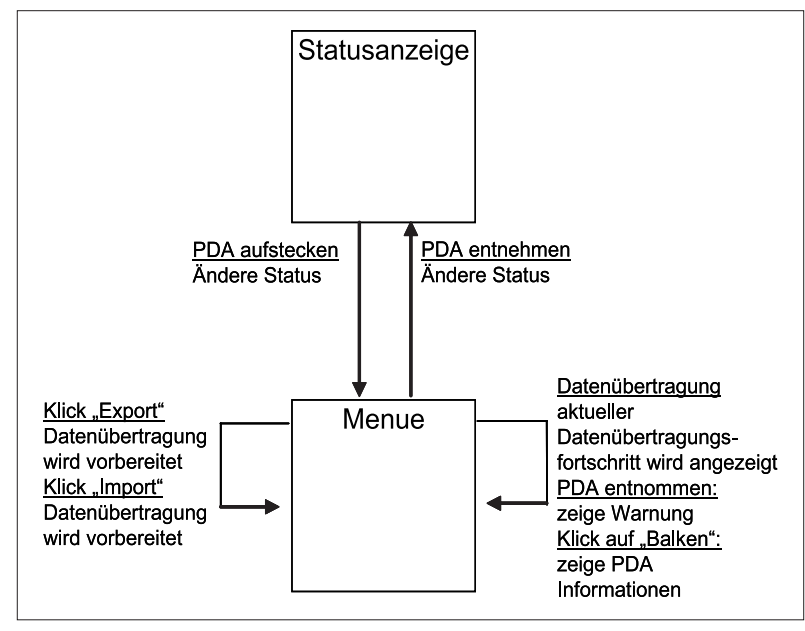

Abb. 4: Zustandsdiagramm der Software

\section{PDA-Software}

Die PDA-Software besteht aus drei Softwarekomponenten. Das GUI-Modul beinhaltet die graphische Benutzeroberfläche. Das StrukturModul ist für die Strukturierung der eingehenden Datenströme, die Datenhaltung in der lokalen PocketPC-Datenbank und den Austausch von Daten zuständig. Das AdminModul erlaubt die Einrichtung von Benutzern und das Festlegen von Berechtigungen und ist durch Passwortschutz nur für den Administrator verfuigbar. Abbildung 5 zeigt das Zusammenspiel dieser Module auf dem PDA.

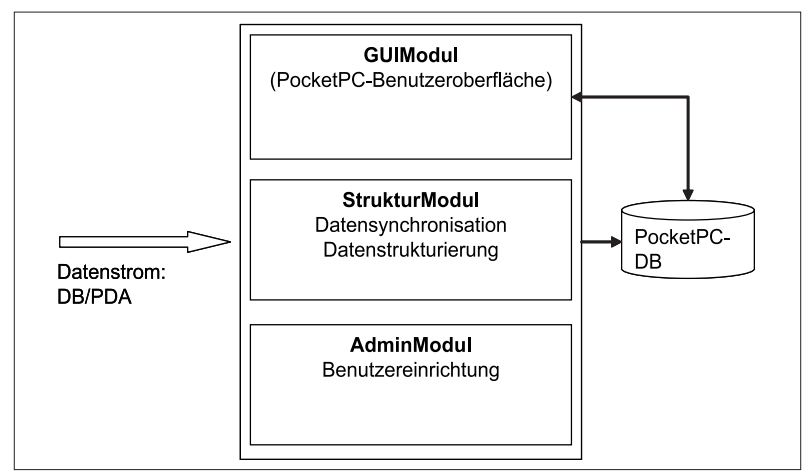

Abb. 5: Softwarestruktur auf dem mobilen Gerät

\subsubsection{Datenbankschnittstelle}

Die Datenbank des Warenwirtschaftssystems beinhaltet alle notwendigen artikel-, paket- und lieferungsbezogenen Daten. Um auf diese zugreifen zu können, war die Entwicklung einer entsprechenden Schnittstelle zur Anbindung des Applikationsservers an das Hostsystem der Firmendatenbank erforderlich. Die Kommunikation erfolgt uiber das Netzwerk (LAN) via TCP/IP. Die Schnittstelle legt außerdem fest, welche Daten aus-/eingelesenen werden sollen. Sie kann beliebige Daten annehmen und von der Datenbank zur Applikation verschicken. Die Daten selbst werden über ASCII-Dateien aus der Datenbank importiert bzw. in die Datenbank exportiert.

\subsection{Benutzeroberflächen}

\subsubsection{Synchronisations-/Dispositionssoftware}

Für die Bedienung am PC musste eine grafische Benutzeroberfläche realisiert werden. Der Anwender sollte mit der Software in der Lage sein,
- sich anzeigen zu lassen, wie viele und welche PDAs an dem System ein- bzw. ausgeloggt sind (Statusanzeige der ein- bzw. ausgeloggten PocketPCs),

- den Datenimport und -export vom/zum PDA zu starten und zu überwachen.

Die grafische Bedienoberfläche wurde mit Java Server Pages (JSP) auf dem Applikationsserver realisiert und wird von dort im HTML-Format zum Internetbrowser übertragen. Die Datenstrukturen (XSD) und die Inhalte wurden in XML festgelegt. Die Darstellung wird durch die Anbindung an die Datenbank aktualisiert.

Ein Auszug aus der Datenstruktur wird in Abb. 6 als Baumstruktur dargestellt.

\begin{tabular}{|c|c|}
\hline Tree Stucluse & Volvest \\
\hline E<rst xadichams & 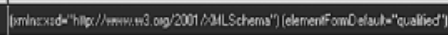 \\
\hline -D [CONMENT] & |Eingetundene Schemsla I \\
\hline ostinctude & [schenolocestion="courniliet xeff"] \\
\hline sestinctude & 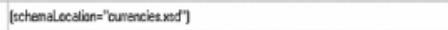 \\
\hline <s xstinclude & (schenalocation="punitxd") \\
\hline D [COMAENT] & Dotenypen I \\
\hline EOt xat simpleType & [naner" dt bodean"] \\
\hline EQt xndrestriction & (bose-"usutsting") \\
\hline Lo madpottan & 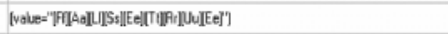 \\
\hline 日)\$ xst simpletype & [name="d_courk'] \\
\hline E-e sastetisition & [base="'natritiege'] \\
\hline L\& satminincivive & [value:"U] \\
\hline E《t xatamplippe & [naneo" dt:punber] \\
\hline Les netreatiction & |basec" aad decinal) \\
\hline$-D[$ [CONMENT] & 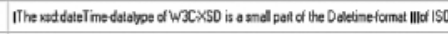 \\
\hline E) xedsimpleTpes & [nane:"d_doteine'] \\
\hline E- E⿺ wediestiction & (basee" "adststing") \\
\hline L\& natpattan & 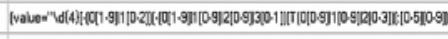 \\
\hline Det xudsimpeTypo & 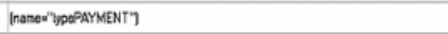 \\
\hline E. 4 netretuition & (basen" "adt:tting") \\
\hline \& sodmexlengh & (vaived"250") \\
\hline \& notminlenglh & [vaver't"] \\
\hline Pot xndsimpetipot & [name-"UpeAdD_INTER"] \\
\hline 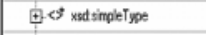 & [name-'Vp:FONE'] \\
\hline 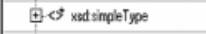 & [nane="'VpeOETAAL"] \\
\hline Tes xat impletype & [name"'TpeSPECONL_TREATMENT_OASS'] \\
\hline$D$ [CONGENT] & 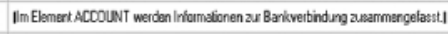 \\
\hline (1) & [name"ACCOUNT"] \\
\hline D [COMENT] & 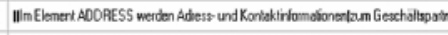 \\
\hline 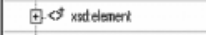 & [nane="ADOAESS"] \\
\hline -D [COMAENT] & 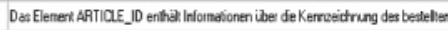 \\
\hline 1] 5 & 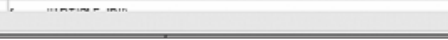 \\
\hline
\end{tabular}

Abb. 6: Auszug der Datenstruktur der Synchronisationssoftware

Die folgenden Screenshots illustrieren das Erscheinungsbild der Anwendungssoftware, bei deren Entwicklung eine intuitive Bedienung im Vordergrund stand.

\section{Dialog: Status PDA}

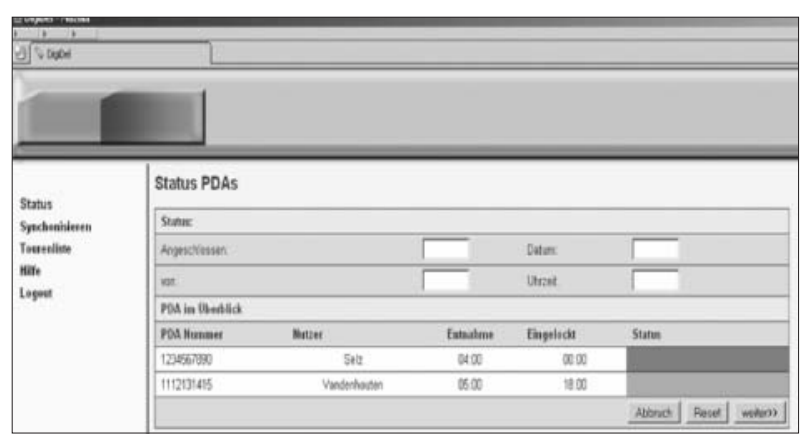

Nach Aufruf des Menüpunktes Statusanzeige erscheint der Dialog „PDA-Status“. Er stellt den Einstieg in den DatenÜbertragungsassistenten dar, der den Anwender durch den gesamten Prozess der Datenübertragung leitet. 


\section{Dialog: Datenimport/Datenexport}

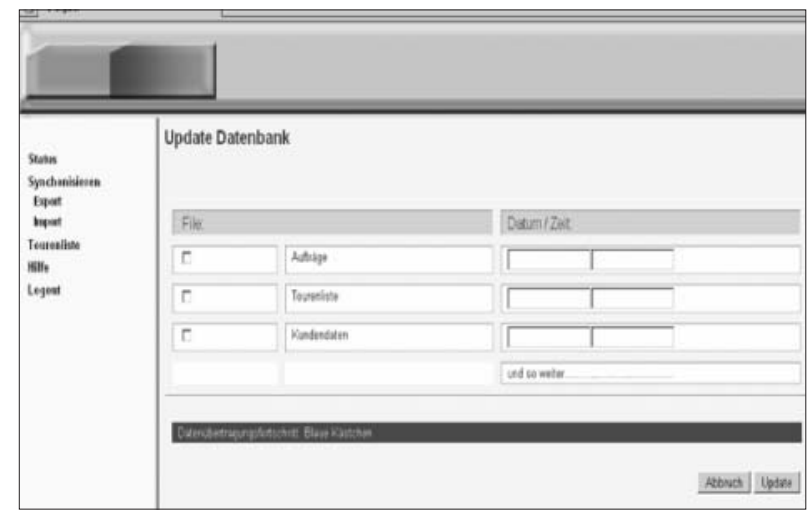

Bevor dieses Fenster erscheint, hat der Nutzer die Möglichkeit, zu entscheiden, ob er Daten exportieren oder importieren möchte. Hier wird die Funktion Datenexport aufgerufen. Nun kann eingestellt werden, welche Daten exportiert werden sollen.

\section{Dialog: Update Datenbank}

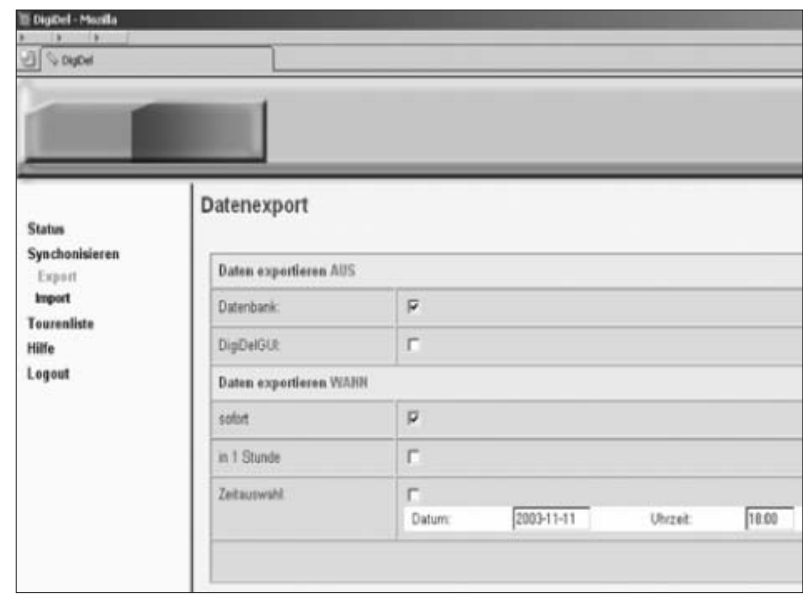

Ein blauer Balken zeigt den Fortschritt des Updates an. Ist der Synchronisationsvorgang abgeschlossen, so befinden sich die Daten, je nach Auswahl, entweder in der Datenbank zur weiteren Archivierung oder auf dem PDA.

\subsubsection{PDA Software}

Mit diesem Modul sollte der Anwender in der Lage sein,

- Pakete und Dokumente zu scannen,

- manuelle Eingaben zu tätigen,

- sich jederzeit Informationen (Tour, Lieferung etc.) auf dem Mobilgerät anzeigen zu lassen,

- Daten, wie z.B. Kundendetails oder die Unterschrift, hinzufügen zu können.

Der Entwicklung der Software in der objektorientierten Sprache Java gingen folgende vorbereitenden Techniken voraus: Es wurde mit der Erstellung eines allgemeinen OOA-Klassendiagrammes (OOA: Objektorientierte Analyse) in UML-Notation (UML: Unified Modeling Language) begonnen. Ziele der Analyse sind die Ermittlung und Beschreibung der Wünsche und Anforderungen des Auftraggebers an das Softwaresystem und die Erstellung eines Modells des Fachkonzepts, das konsistent, vollständig, redundanzfrei und realisierbar ist (siehe Abb. 8).
In der OOA wurde das Problem analysiert und so aufbereitet, dass es nun im OOD-Modell (Objektorientiertes Design) für die Hard- und Softwareplattformen weiter verfeinert werden konnte. Der Vorgang umfasste das Design einer Benutzeroberflächen-, einer Verarbeitungsund einer Datenmanagementkomponente. Letztere ist in Abb. 9 zu sehen.

Die Screenshots in Abb. 10 zeigen exemplarisch die grafische Benutzeroberfläche des PDA. Bei der Entwicklung wurde besonders darauf geachtet, dass überwiegend eine Bedienung mit dem Finger möglich ist. Farbige Anzeigen geben eine schnelle Auskunft über getätigte Vorgänge (z.B. Fehler beim Scannen: rotes Feld leuchtet auf).

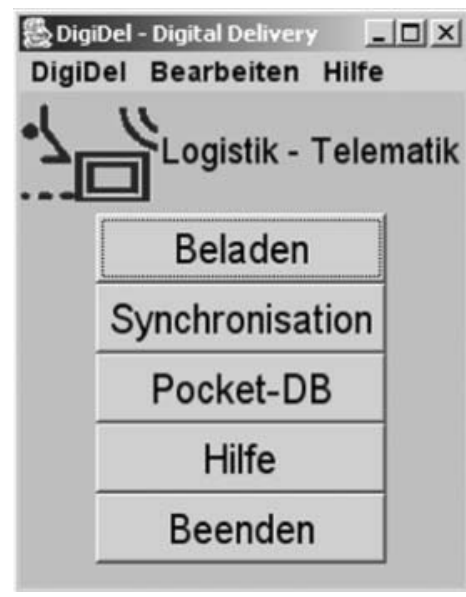

Abb. 10 a: Hauptmenü

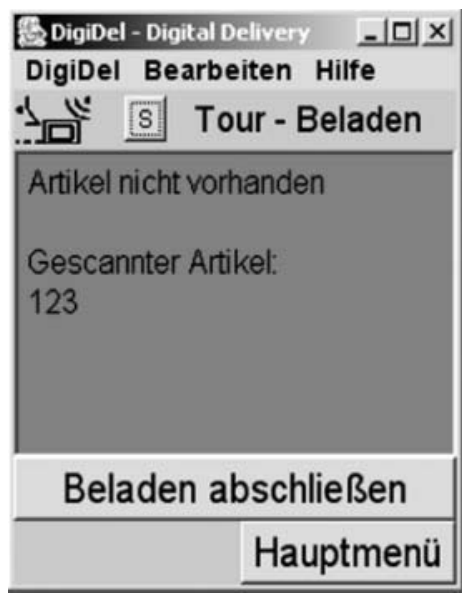

Abb. 10 b: Fehler beim Scannen, rotes Feld

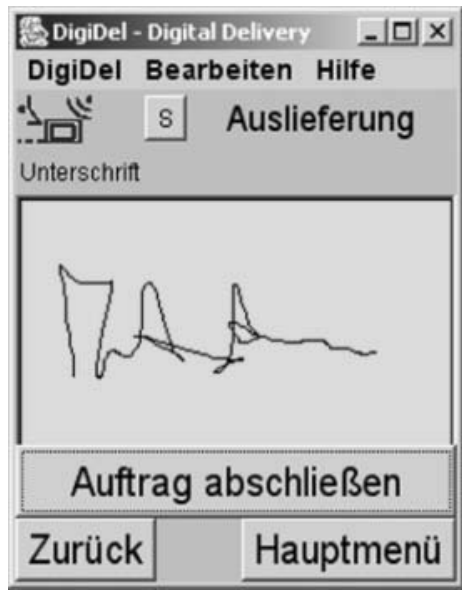

Abb. 10 c: Unterschrifterfassungsfeld 


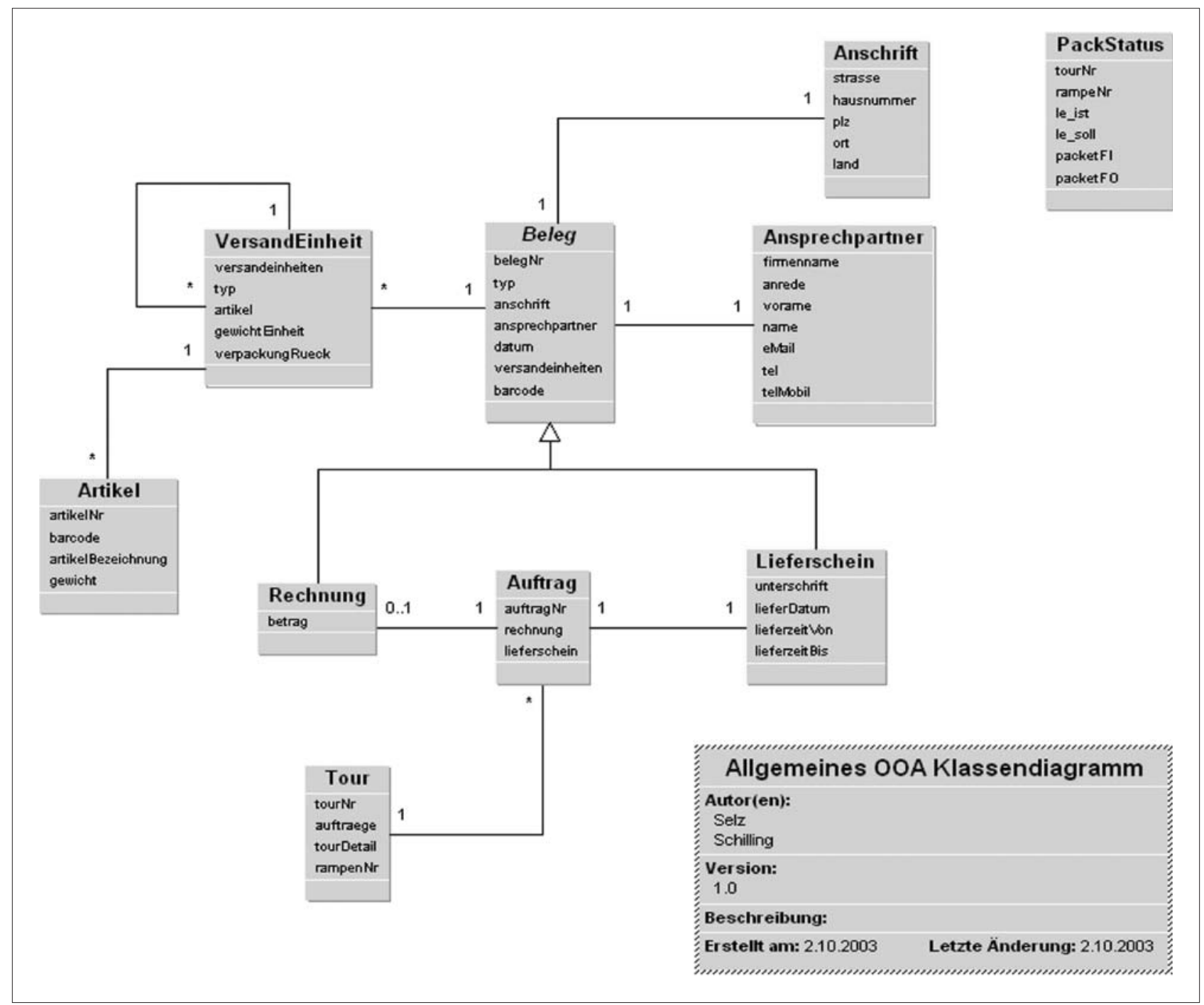

Abb. 8: OOA-Modell für die Datenhaltung im PDA

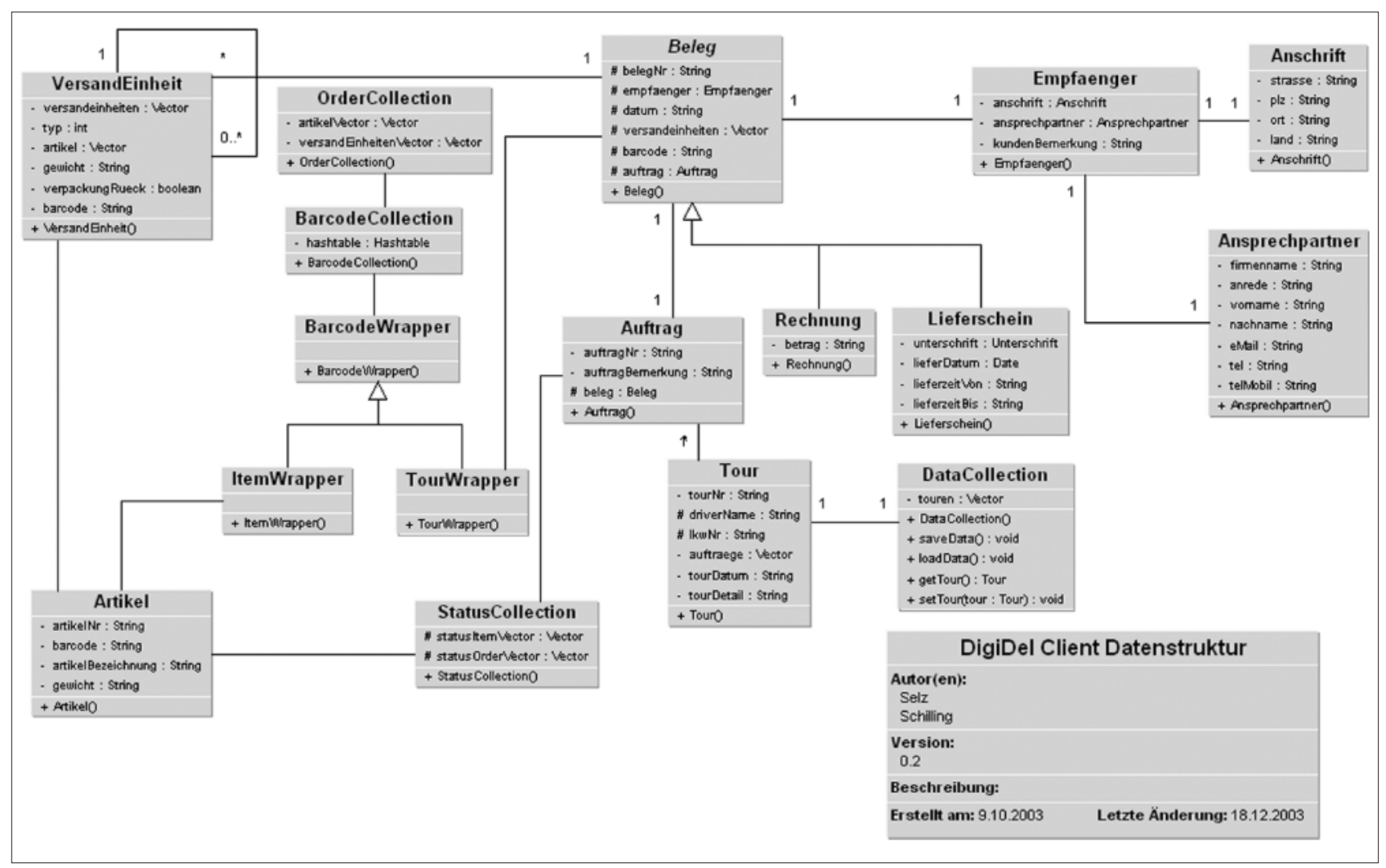

Abb. 9: OOD-Modell des Datenmanagements auf dem PDA 


\section{Technologietransfer}

Das im Rahmen einer Bachelorabschlussarbeit spezifizierte und auf der CeBit 2003 erstmalig demonstrierte Projekt wurde inzwischen im Rahmen eines Drittmittelprojektes von einem mittelständischen Unternehmen angenommen und kann als Beispiel für die erfolgreiche Kooperation zwischen Wissenschaft und Wirtschaft dienen. Die vollständige Implementierung soll bis Dezember 2004 abgeschlossen sein.

\section{Literatur}

Helmut Balzert: Lehrbuch der Software-Technik, 2 Bde., 1) Software Management, 2) Software-Entwicklung, 2. Auflage, Spektrum Akademischer Verlag, Heidelberg 2000.

Henning Behme, Stefan Mintert: XML in der Praxis, AddisonWesley Verlag, München 2000.

Rüdiger Hossner (Hrsg.): Logistik Jahrbuch 2003, Verlagsgruppe Handelsblatt Fachverlag, Düsseldorf 2003.

Hans-Christian Pfohl: Logistikmanagement. Funktionen und Instrumente (Logistik in Industrie, Handel und Dienstleistungen), Springer Verlag, Berlin u. a. 1994.

Westy Rockwell: XML, XSLT, Java und JSP. Professionelle WebApplikationen entwickeln, Galileo Computing/Galileo Press $\mathrm{GmbH}$, Bonn 2000.

http://www.w3.org/

http://www.jsp-develop.de/

http://www.symbol.com

http://www.logistik-heute.de/

http://www.verband-lb.de/

http://www.dvz.de/

\section{Autoren}

Prof. Dr. rer. nat. Ralf Vandenhouten

Technische Fachhochschule Wildau

Fachbereich Ingenieurwesen/Wirtschaftsingenieurwesen

Lehrstuhl für Telematik

Tel. +493375 508-359

E-Mail: rvandenh@igw.tfh-wildau.de

Miriam Selz, B.Eng.

Technische Fachhochschule Wildau

Fachbereich Ingenieurwesen/Wirtschaftsingenieurwesen Lehrstuhl für Telematik

Tel. +493375 508-616

E-Mail: mselz@tfh-wildau.de 